Western University Scholarship@Western

Centre for the Study of International Economic

Centre for the Study of International Economic

Relations Working Papers

Relations

1980

\title{
A Decomposition Algorithm for General Equilibrium Computation with Application to International Trade Models
}

Ahsan Mansur

John Whalley

Follow this and additional works at: https://ir.lib.uwo.ca/economicscsier_wp

Part of the Economics Commons

Citation of this paper:

Mansur, Ahsan, John Whalley. "A Decomposition Algorithm for General Equilibrium Computation with Application to International Trade Models." Centre for the Study of International Economic Relations Working Papers, 8004. London, ON: Department of Economics, University of Western Ontario (1980). 
CENTRE FOR THE STUDY OF INTERNATIONAL ECONOMIC RELATIONS

WORKING PAPER NO. 8004

A DECOMPOSITION ALGORITHM FOR GENERAL EQUILIBRIUM COMPUTATION WITH APPLICATION TO INTERNATIONAL TRADE MODELS

\author{
Ashan Mansur \\ and \\ John Whalley
}

This paper contains preliminary findings from research still in progress and should not be quoted without prior approval of the author.

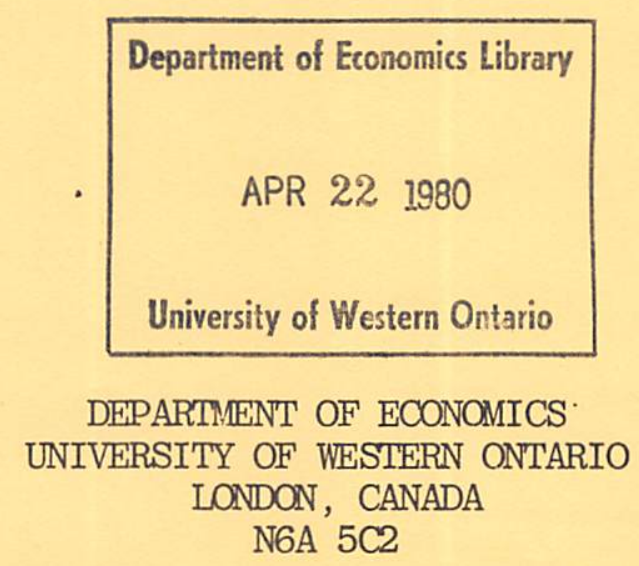




\title{
A DECOMPOSITION ALGORITHM FOR GENERAL EQUILIBRIUM COMPUTATION WITH APPLICATION TO INTERNATIONAL TRADE MODELS
}

by

\author{
Ahs.an Mansur \\ and \\ John Whalley \\ Department of Economics \\ University of Western Ontario \\ London \\ Canada
}

March 1980

$1_{\text {The second author acknowledges financial support from the Ford }}$ Foundation under their programme of research on the International Economic Order. 


\section{Introduction}

In this paper we describe the computation of general equilibrium via a fixed point decomposition procedure similar in spirit to the Dantzig-Wolfe decomposition algorithm for the solution of linear programming problems [Dantzig and Wolfe (1961)]. We show that for a general equilibrium model of a particular structure it is possible to compute equilibria using 'master' and 'sub' simplices each of a dimensionality smaller than that of the total problem. The analogues to the common constraints in the Dantzig-Wolfe procedure are common commodities with common prices, and the block diagonal structure on non-common constraints is replaced by an analogous block diagonal pattern of demands and endowments of agents over non-common goods. The procedure is guaranteed to terminate at an approximate equilibrium without cycling by the same argument underlying Scarf's algorithm. In the case of a pure trade equilibrium we use variations on the traditional Gale Nikaido mapping for label generation but use dimensions smaller than that of the whole problem. A natural application of the method is to international trade models with 'traded' and 'non-traded' goods. We use this example for illustration but regional and intertemporal models would also appear to offer fruitful areas of application.

The method involves the generation of labels for vertices on a master simplex through the separate solution of sub-equilibrium problems whose parameters are determined by the vertex on the master simplex. Information is passed between a master problem and sub problem as in the Dantzig-Wolfe algorithm; 'coefficlent generation' for a master linear programming problem in the Dantzig-Wolfe algorithm is replaced by 'label generation' for vertices on a 'master' simplex associated with the general equilibrium problem. We have performed computation with this method for some 
numerical examples using Merrill's algorithm for solution of both full

dimensional problems and the same problems by the decomposition procedure. A quicker procedure for large models would be to use the recent methods of vander Laan and Talman (1979), but for comparative purposes the consistent application of Merrili's algorithm would appear to be adequate. Where a number of blocs of congumer groups with only partially overlapping excess demand functions are involved significant computational gains are indicated, suggesting eventual possible application to empirically oriented large-scale general equilibrium models of world trade. At least two models in current use have the same structure of common and non-common goods as examined here; referred to as 'traded' and 'non-traded' goods in the international trade 1iterature. In one Deardorff and Stern (1979)] 18 separate countries appear and in the other [Whalley (1979)] 4 major trading areas are identified. Our discussion is exclusively in terms of integer labeling problems; we believe the extension to vector labelling problems follows naturally but have not extensively explored the issue.

The economic interpretation of our procedure is that we decompose the list of commodities in a general equilibrium problem into 'common' goods traded among all agents and 'non-common' goods traded only among a subset of agents. The allocation of non-common goods to agents can be represented in a bloc diagonal partition of demands and asset ownership by agent. We use a master simplex containing information on the prices of common goods. The subequilibrium problems use information on the relative prices of common goods from the master simplex to form a vector of prices for both common goods and non-common goods in the bloc. Each sub-equilibrium problem takes the relative prices of common goods as given and determines an approximate equilibrium characterized by non-positive excess demands for each non-common good along with an equilibrium price for a composite common good meeting 
a non-negative excess demand condition. The solution for subproblems together with the common good prices on the master simplex yield an evaluation of excess demand functions for common goods which generates a label for a vertex on the master simplex. A completely labelled master simplex along with associated sub-equilibria yield an approximation to an equilibrium for the whole model, which becomes exact in the limit approached by a dense grid. Each subproblem may be interpreted as an equilibrium problem for a small open economy with non-traded goods, which takes relative prices of traded goods as given by world markets and determines an equilibrium in which demand supply equalities hold for non-traded goods along with an external sector balance condition. The demonstration of existence of equilibrium for such a trade model does not appear to be in the literature, but would seem to follow directly from the formulation of sub-equilibrium problems we present. An alternative interpretation is that we partition the list of commodities in the model so that we can apply the Hicks-Leontief composite commodity theorem. 1 We construct a composite common good for an equilibrium problem for each bloc involving the single composite common good and all non-common goods specific to that bloc. 'Sub'equilibrium solutions are communicated back to the 'master' simplex and used in such a way that 'master' solution implies full solution.

II. A Pure Exchange General Equilibrium Model with a Decomposition Structure Between Common and Non-Common Goods

We consider a pure exchange general equilibrium model where each agent has a fixed endowment of common goods and certain of the non-common goods. The decompositon structure we stress is that common goods are both demanded and owned initially by all agents while non-common goods are owned and demanded by only a subset of agents.

This theorem is discussed in Arrow and Hahn [1971] pp. 6-8. 
The economy consists of $\mathrm{K}$ groups of agents each characterized by (i) a set of demand functions and (ii) a vector of endowments (of both common and non-common goods). Market demand functions for the non-common goods are the sum of the individual demands only within the group involved. Market demand functions for the common goods are given by the sum of demand functions across all agents. The common goods are indexed $1, \ldots, n_{0}$; the non-common goods indexed from $n_{0}+1, \ldots, n_{1}($ bloc 1$) ; n_{1}+1, \ldots, n_{2}$ (bloc 2),... and so on. The model has a total of $N=n_{0}+\sum_{i=1}^{K}\left(n_{i}-\left(n_{i-1}+1\right)\right)$ goods. We consider a price vector $\pi$ of dimensionality $N$ which contains prices of all goods. $w_{i}^{k}$ represents the endowment of good $i$ by group $k$. We assume $w_{i}^{k} \geq 0$ and $>0$ for some $i$ for each $k$; in addition $w_{i}^{k}=0$ for $i=n_{\ell-1}+1, \ldots, n_{\ell}$ and $l \neq k$ (zero endowments in group $k$ of non-common goods of other groups of agents).

The market demands by group $k$ for the common goods, $1, \ldots, n_{0}$, and non-common goods relevant to the group, $n_{k-1}+1, \ldots, n_{k}$, are functions only of the corresponding commodity prices $\left(\pi_{1}, \ldots, \pi_{n_{0}}, \ldots, \pi_{n_{k-1}}+1, \ldots \pi_{n_{k}}, \ldots\right)$. We will use this property later but for convenience write these functions as $\xi_{i}^{k}(\pi)$; the demands for good $i$ by group $k$. The demand functions for each group are assumed to be non-negative, continuous, homogeneous of degree zero in all prices, and to satisfy a version of Walras' Law defined for that group alone. This implies that total demand functions satisfy a model wide version of Walras' Law. The zero homogeneity of demands allows prices of all common and non-common goods in the economy to be normalized to sum to any non-negative constant; we work with a price simplex all of whose vectors contain coordinates with sum $D ; \sum_{i=1}^{N} \pi_{i}=D$. An equilibrium in this model is a vector of prices $\pi^{*}$ such that all excess demands are non-positive with zero prices prevailing for any commodity with strictly negative excess demands. These conditions may be written out explicitly as 


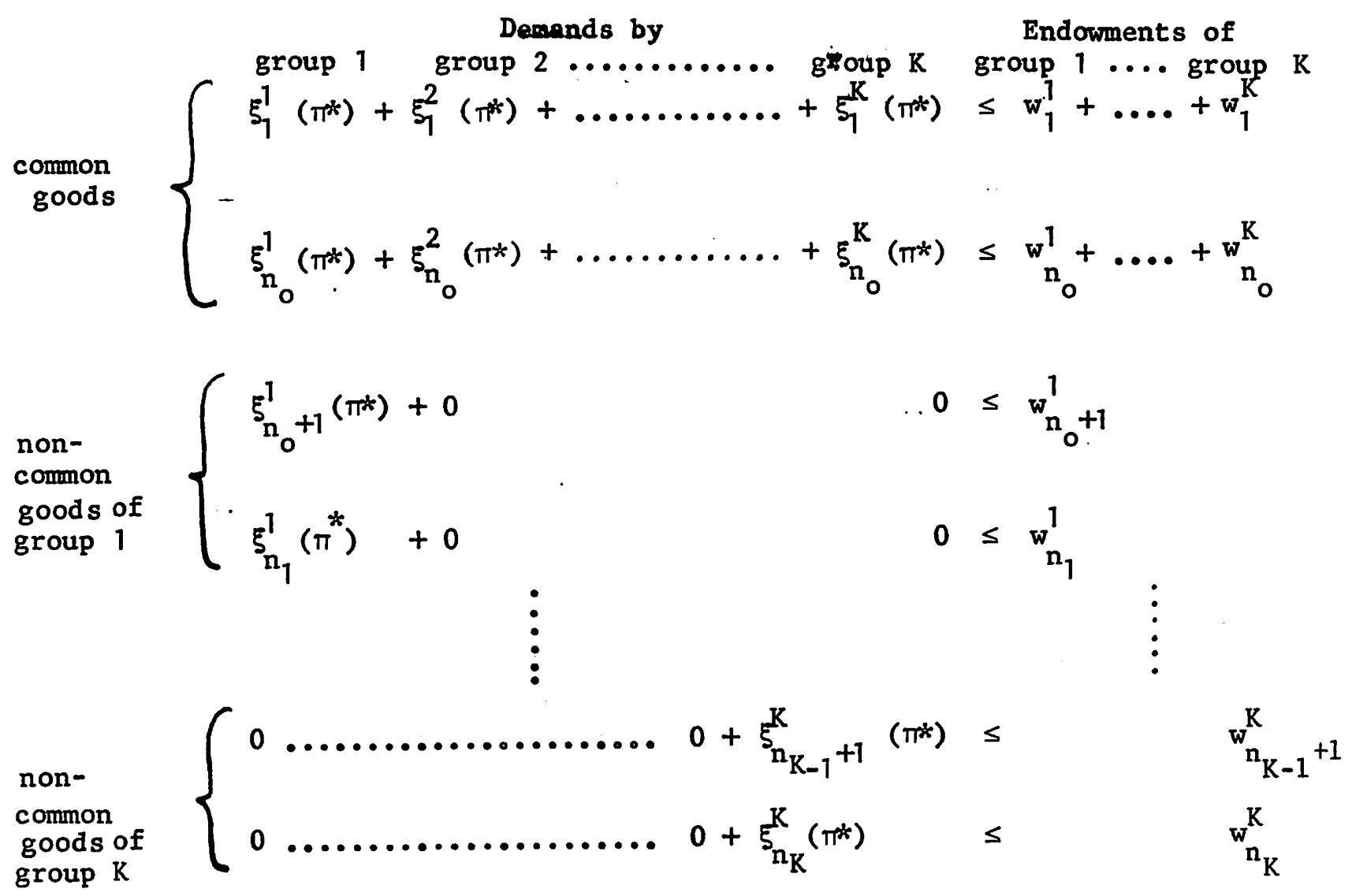

with the corresponding $\pi_{i}^{*}$ equalling zero if any inequality holds strictly. The statement of equilibrium conditions above is visually similar to the constraint matrix in the Dantzig-Wolfe decomposition algorithm where a set of common constraints prevailswith the remainder of the constraint matrix being written in block diagonal form. Here a similar structure applies to both demands and initial endowments when subscripted by group. One can clearly use Scarf's (1973) algorithm (applying one of the recent refinements to reduce execution times such as vander Laan and Talman (1979) and Shamir (1979)) directly to this $\mathrm{N}$-dimensional model ignoring its special structure. Our interest is in a computational method using a lower dimensionality than $\mathrm{N}$ which takes advantage of this special structure while still preserving the Lemke-Howson no-cycling argument used by Scarf's algorithm. 
III. A Decomposition Algorithm for Equilibrium Computation

In the Dantzig-Wolfe decomposition procedure a full dimensional linear programming problem with common constraints and a bloc diagonal structure for other constraints is rewritten as an equivalent problem requiring only one constraint for each of the diagonal blocs in the constraint matrix of the original problem. This problem involves all the (unknown) vertices associated with the constraint set for each of the diagonal blocs in the non-common portion of the constraint set. Columns are generated for the simplex tableau of the reduced dimension equivalent problem by solving sub LP problems generating a vertex for each bloc with the smallest entry for the objective function in the tableau. Vertices are successively added through the column generating procedure which sub problem solutions provide. Pivot steps in the master tableau in turn provide coefficients for the objective function in sub problems. When all sub problems produce non-positive solutions, all tableau entries for coefficients in the objective function associated with vertices of the constraint sets are non-negative and a maximum to the linear programming problem must have been determined. The method allows a large dimensional linear programming problem to be solved through a sequence of smaller dimensional problems without any necessary requirement that all vertices describing constraint sets for diagonal blocs be evaluated. The no-cycling argument in the traditional form of the simplex method is preserved.

In our fixed point decomposition procedure, we first rewrite the general equilibrium conditions above as two interdependent sets of conditions which if they jointly hold at the same set of prices ensure equilibrium. Our procedure Involves passing information between a master simplex to sub simplices until both sets of conditions are satisfied. The labelling rule used for vertices on the master simplex guarantees that in the limit associated with a dense grid being 
used to solve both master and subproblems the same price vectors must occur at the two solution sets.

Our equivalent statement of equilibrium conditions involves rewriting the price vector $\pi$ as $\left(\pi^{c}, \pi^{1}, \ldots, \pi^{K}\right)$ where $\pi^{c}$ refer to prices of common goods and $\pi^{1}, \ldots, \pi^{K}$ are the prices of non-common goods for each group. We note that the demand functions $\xi_{i}^{k}(\pi)$ of group $k$ can be rewritten as $\xi_{i}^{k}\left(\pi^{c}, \pi^{k}\right)$.

We partition the equilibrium conditions, written in full above, and characterize a 'common good' equilibrium (Master Equilibrium) as a vector $\pi^{*}=\left(\pi^{c^{*}}, \pi^{1 *}, \ldots, \pi^{K^{*}}\right)$ such that excess demands are non-positive for each common good, i.e.:

$$
\sum_{k}\left(\xi_{i}^{k}\left(\pi^{c^{*}}, \pi^{k^{*}}\right)-w_{i}^{k}\right) \leq 0 \quad\left(=0 \text { if } \pi_{i}^{c^{*}}>0\right) \quad i=1, \ldots, n_{0}
$$

'Non-common' good equilibria (Sub Equilibria) are characterized in terms of a vector $\left(\pi^{c^{*}}, \pi^{k^{*}}\right)$ for any group $k$ such that excess demands are non-positive for each non-common good, i.e.

$$
\xi_{i}^{k}\left(\pi^{c^{*}}, \pi^{k^{*}}\right)-w_{i}^{k} \leq 0 \quad\left(=0 \text { if } \pi_{i}^{k *}>0\right) \quad i=n_{k-1}+1, \ldots, n_{k} \cdot
$$

We note that from Walras' Law and the property that non-common goods cannot be traded across groups, the yalue of excess demands for common goods

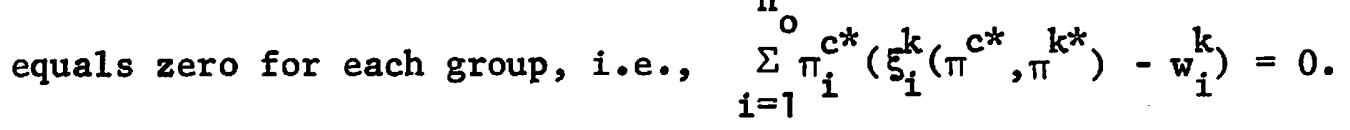

If each of these sets of equilibrium conditions simultaneously holds at the same sets of prices then full equilibrium must prevail. While it may seem an indirect procedure to partition the equilibrium conditions in order to characterize two 'partial' equilibria which jointly imply the general equilibrium conditions a potential computational saving is suggested by this device. If, in some way, the common goods prices can be assumed to be fixed to solve for sub-equilibria and the non-common goods prices calculated in this way used in determining master equilibria, a procedure which passes 
information between alternative equilibrium problems offers a possibility of a solution method which exploits the special bloc diagonal structure of excess demand functions. The Hicks-Leontief composite commodity theorem suggests that aggregating quantities within a partition yields a composite commodity which can be analytically treated as equivalent to a single commodity. We exploit this by arguing that given any fixed relative prices for common goods, there exist prices for the composite commodity and noncommon goods for the bloc that satisfy non-positive excess demand conditions. We thus solve sub-equilibria using the composite commodity and communicate prices of non-common goods back to the master simplex for an evaluation of excess demands of common goods. By reducing the dimensionality of each problem in this way, the hope is that by determining subsets of equilibrium prices with equilibrium holding for a composite of the common goods a complete equilibrium can be determined and yield computational gains over full solution. Our method shows that this is possible and initial computational experience suggests that computational gains of some potential significance are possible.

\section{Sketch of the Procedure}

Taking a simplex of full dimensionality containing all $\mathrm{N}$ commodity prices $\pi_{i},(i=1, \ldots, N)$, we reduce the dimensionality of this simplex to $n_{0}$ by retaining only the prices of common goods. We term this the master simplex.

Using a regular grid to represent the subdivision of the master simplex, we separately construct a simplex on which we search for a sub-equilibrium for each group where simplices for sub problems are all associated with the vertex of the subdivision of the master simplex from which they are derived. These associated simplices are each of dimension equal to the number of non-common goods for the group concerned plus one. For convenience, all the coordinate sums for vertices on associated simplices are taken to be equal to that of vertices on the master simplex. 
We compute 'sub-equilibria' for each group using these simplices and assuming the relative prices of common goods from the vertex on the master simplex to be fixed. The equilibrium conditions in these problems are limited to the non-positive excess demand for the composite of common goods constructed using the relative common goods prices from the master simplex, rather than requiring each excess demand to be non-positive as in full equilibrium. In any sub-equilibrium we thus determine inarket clearing prices of non-common goods and a scalar multiple for all common goods prices giving non-positive excess demands for the composite common good using the fixed relative prices from the vertex on the master simplex as weights.

We return our sub-solution to the vertex on the master simplex and list the prices of non-common goods along with the common goods prices. We use the derived price vector to evaluate excess demand functions, and our labelling rule for any vertex on the master simplex involves the excess demands for common goods. A completely labelled simplex of dimension $n_{0}$ in the subdivision of the master simplex (along with the associated approximate sub-equilibria) will characterize an approximation to an equilibrium for the entire general equilibrium system.

\section{Description of 'master' and 'sub' simplices}

We define the master simplex of dimension $\mathrm{n}_{0}$ as $\mathrm{s}_{0}$ and consider a subdivision of this simplex to be represented by the vertices $s_{0}^{j}$. Each vertex $s_{0}^{j}$ will be written as

$$
s_{0}^{j}=\left[\begin{array}{l}
\pi_{1}^{j} \\
\vdots \\
\pi_{n}^{j}
\end{array}\right] ; \quad \begin{aligned}
& \sum_{0=1}^{n} \pi_{i}^{j}=D \\
& \text { for all } j .
\end{aligned}
$$


For convenience, we will restrict ourselves to subdivisions of $S_{0}$ characterized by a regular grid with mesh size given by the integer $D$.

Corresponding to each vertex $s_{0}^{j}$, we also define $K$-simplices $s_{1}^{j}, s_{2}^{j}, \ldots, s_{K}^{j}$ (one corresoonding to each group) such that the sums of coordinates on those simplices are also given by $D$. We consider simplicial subdivisions of each simplex $\mathrm{s}_{1}^{\mathrm{j}}, \ldots, \mathrm{s}_{\mathrm{K}}^{\mathrm{j}}$ in addition to the subdivision of $\mathrm{s}_{\mathrm{o}}$ represented by the vertices $s_{0}^{j}$. The $h^{\text {th }}$ vertex of the subdivision of the $k^{\text {th }}$ simplex $s_{k}^{j h}$ is written as

$$
s_{k}^{j h}=\left(\begin{array}{c}
\pi_{n_{k-1}+1}^{j h} \\
\vdots \\
\pi_{n_{k}}^{j h} \\
c_{k}^{j h}
\end{array}\right)
$$

where $k=1,2, \ldots, k$ and the superscript $j$ corresponds to the $j^{\text {th }}$ vertex $s_{o}^{j}$.

\section{Characterization of Sub-Equilibrium Problems}

Corresponding to any vertex $\mathrm{s}_{k}^{j h}$ in a subdivision of $\mathrm{s}_{k}^{j}$ we determine a vector of prices of common and non-common goods using the relative prices of common goods $\pi_{1}^{j}, \ldots, \pi_{n_{0}}^{j}$ from $s_{o}^{j}$, the sum of common goods prices from $s_{k}^{j h}$, and the prices of non-common goods $\pi_{n_{k-1}}^{j h}+1, \cdots, \pi_{n_{k}}^{j h}$ from $s_{k}^{j h}$. We represent this by the function $\tilde{\pi}_{k}^{j h}\left(s_{o}^{j}, s_{k}^{j h}\right)$ where

$$
\tilde{\pi}_{k}^{j h}\left(s_{o}^{j}, s_{k}^{j h}\right)=\left(\frac{\pi_{1}^{j} \cdot c_{k}^{j h}}{D}, \frac{\pi_{2}^{j} \cdot c_{k}^{j h}}{D}, \ldots, \frac{\pi_{n_{0}}^{j} \cdot c_{k}^{j h}}{D}, \pi_{n_{k-1}+1}^{j h}, \ldots, \pi_{n_{k}}^{j h}\right) .
$$


The function $\tilde{\pi}_{k}^{j h}\left(s_{0}^{j}, s_{k}^{j h}\right)$ determines prices of both common and non-common goods for group $k$ where the relative prices of common goods are consistent with corresponding values from the vertex $\mathrm{s}_{0}^{j}$ on the master simplex. The relative prices of the noncommon goods are given by the vertex $\mathrm{s}_{k}^{j h}$ on the simplex associated with the subproblem. The sum of the prices of common goods is constrained to equal $c_{k}^{j h}$, the last element of the vector defining the vertex $\mathrm{s}_{k}^{j h}, \mathrm{c}_{k}^{j h}$ can be zero in which case the terms

$\frac{\pi_{1}^{j} \cdot c_{k}^{j h}}{D}, \ldots, \frac{\pi_{n_{0}}^{j} \cdot c_{k}^{j h}}{D}$ are all zero.

For any group $k$, a 'sub-equilibrium problem' is constructed whose equilibrium solution is defined as a vector $\tilde{\pi}_{k}^{j *}\left(s_{o}^{j}, s_{k}^{j *}\right)$ associated with a vector $s_{k}^{j *}$ on the simplex $\mathrm{s}_{\mathrm{k}}^{\mathrm{j}}$ such that

$$
\text { (ii) } \sum_{i=1}^{n_{0}} \frac{\pi_{i}^{j}}{D}\left(\xi_{i}^{k}\left(\tilde{\pi}_{k}^{j *}\right)-w_{i}^{k}\right) \leq 0 \quad\left(=0 \text { if } G_{k}^{j *}>0\right) \text {. }
$$

In this problem common goods are aggregated at the fixed prices $\pi_{i}^{j}$. Equilibrium prevails when both non-positive excess demands summed across all consumers in group $k$ occur for the composite of common goods and for each non-common good. $c_{k}^{j *}$ can be interpreted as the equilibrium price for the composite of common goods (constructed using the fixed relative prices $\pi_{1}^{j}, \ldots, \pi_{n_{0}}^{j}$ as weights) relative to each of the non-common goods for group $k$.

\section{Solution of Sub-Equilibrium Problems}

Using the vector function $\tilde{\pi}_{k}^{j h}\left(s_{0}^{j}, s_{k}^{j *}\right)$ for any vertex $s_{k}^{j h}$ we can apply Scarf's algorithm (or one of the recent extensions mentioned earlier) to compute an approximation to a sub-equilibrium $s_{k}^{j *}=\left(\pi_{n_{k-1}}^{j^{*}}, \cdots, \pi_{n_{k}}^{j^{*}}, c_{k}^{j *}\right)$.

This provides an approximation to an equilibrium to the $k^{\text {th }}$ sub-problem which is used in the labelling procedure for the vertex $\mathrm{s}_{0}^{j}$ on the master simplex in a way to be described later. 
The equilibrium computation differs in the sub-problems from the application of Scarf's algorithm to a standard exchange economy only in a small modification in the use of the Gale-Nikaido mapping to determine labels for vertices on each simplex $\mathrm{s}_{k^{j}}^{j}$. The conventional labeliling procedure for an $N$ commodity exchange equilibrium problem defined on an $\mathrm{N}$ dimensional price simplex calls for selecting as the label to any vertex $\pi^{j}$ the index of the first coordinate for which $f_{i}\left(\pi^{j}\right)-\pi_{i}^{j}>\theta(i=1, \ldots, N)$, where $f_{i}$ define the wellknown Gale-Nikaido transformation of the excess demand functions which produce a continuous mapping of the unit simplex into itself.

In the solution of each sub-problem we use a related mapping of a $\left(n_{k}-n_{k-1}\right)$ dimensional simplex into itself given by

$$
\begin{aligned}
& g_{i}^{k}\left(\tilde{\pi}_{k}^{j h}\left(s_{0}^{j}, s_{k}^{j h}\right)\right)=\frac{\pi_{i}^{j h} / D+\max \left[Q_{3}\left(\xi_{i}^{k}\left(\tilde{\pi}_{k}^{j h}\right)-w_{i}^{k}\right)\right]}{1+\sum_{i=n_{k-1}+1}^{n_{k}} \max \left[0,\left(\xi_{i}^{k}\left(\tilde{\pi}_{k}^{j h}\right)-w_{i}^{k}\right)\right]+\max \left[0, \sum_{i=1}^{n_{0}} \frac{\pi_{i}^{j}}{D}\left(\xi_{i}^{k}\left(\tilde{\pi}_{k}^{j}\right)-w_{i}^{k}\right)\right]} \\
& \text { for } i=n_{k-1}+1, \ldots, n_{k} \\
& c_{k}^{j h} / D+\max \left[0, \sum_{i=1}^{n} \frac{\pi_{i}^{j}}{D}\left(\xi_{i}^{k}\left(\tilde{\pi}_{k}^{j}\right)-w_{i}^{k}\right)\right] \\
& g_{n_{k+1}}^{l k}\left(\tilde{\pi}_{k}^{j h}\left(s_{o}^{j}, s_{k}^{j h}\right)\right)=\frac{1=1}{1+\sum_{i=n_{k-1}+1}^{n_{k}} \max \left[0,\left(s_{i}^{k}\left(\tilde{\pi}_{k}^{j h}\right)-w_{i}^{k}\right)\right]+\max \left[0, \sum_{i=1}^{n_{0}} \frac{\pi_{i}^{j}}{D}\left(s_{i}^{k}\left(\tilde{\pi}_{k}^{j}\right)-w_{i}^{k}\right)\right]}
\end{aligned}
$$

The label of vertex $s_{k}^{j h}$ in the $k^{\text {th }}$ sub-problem is taken as the first $i$ for which the Image $g_{i}^{k}\left(\tilde{\pi}_{k}^{j h}\left(s_{o}^{j}, s_{k}^{j h}\right)\right)$ exceeds one over $D$ multiplied by its corresponding coordinate on $\mathrm{s}_{\mathrm{k}}^{\mathrm{jh}}$. A completely labelled simplex w111 have all the labels $1, \ldots,\left(n_{k}-n_{k-1}\right)$ including a label derived from the transformation of the excess demand for the composite good. The modification to the GaleNikaido transformation incorporates the feature that a composite of excess demands is involved for the common goods. The starting and termination procedures are as in any other application of Scarf's algorithm. 


\section{Iabelling Rules for Vertices on the Master Simplex}

While solutions to sub-equilibrium problems corresponding to a vertex

$s_{0}^{j}$ on the master simplex will yield approximations to the equilibrium conditions required for all non-common goods, they will not typically result in market excess demands for each of the common goods being less than or equal to zero. We therefore construct a labeling rule for any vertex $\mathrm{s}_{0}^{\mathrm{j}}$ on the master simplex such that for a completely labelled simplex in a subdivision of $\mathrm{S}_{0}$ this will be the case. The labels are selected from integers $1, \ldots, n_{0}$ and for $a$ completely labelled simplex an approximation to an equilibrium for the entire general equilibrium problem will be obtained. This approximation becomes exact in the limit as the mesh of vertices defining the subdivision becomes everywhere dense on the simplex $\mathrm{S}_{0} \cdot$

We begin with the solution to the first sub-equilibrium problem and construct the vector

$$
\hat{\pi}^{1 j}=\left(\lambda_{1}^{j} \pi_{n_{0}+1}^{j^{*}}, \ldots, \lambda_{1}^{j} \pi_{n_{1}}^{j^{*}}\right)
$$

where $\lambda_{1}^{j}$ is defined as $\frac{\sum_{i=1}^{n_{0}} \pi_{i}^{j}}{c_{1}^{j^{*}}}$, and $\pi_{n_{0}+1}^{j *}, \ldots, \pi_{n_{1}}^{j *}$ define the sub-equilibrium non-common goods prices from the solution of the first sub-equilibrium problem.

Where a non-dense grid is used for a sub-equilibrium problem the noncommon goods prices $\pi_{n_{0}+1}^{j^{*}}, \ldots, \pi_{n_{1}}^{j^{*}}$ together with $C_{i}^{j^{*}}$ may be taken from the mid point of the completely labelled simplex in the subdivision of the simplex associated with the subproblem. In the finite grid case $c_{1}^{j *}$ cannot equal zero, in the limiting case of a dense grid $\lambda_{1}^{j}$ may approach infinity if $c_{1}^{j *}$ approaches zero. A construction we use in our labelling rule below guarantees that even if $c_{k}^{j^{*}}$ 
approaches zero for $a 11 \mathrm{~K}$ sub-problems, a limit vector on the master simplex with zero common goods prices but some non-zero non-common goods prices will be approached through a convergent subsequence.

Additional vectors $\hat{\pi}^{2 j}, \ldots, \hat{\pi}^{K j}$ can be constructed in a manner similar to that for $A^{1 j}$ using solutions to the other sub-equilibrium problems. The vectors $\hat{\pi}^{1 j}, \ldots, \hat{\pi}^{K j}$ together with the common goods prices $\pi_{1}^{j}, \ldots, \pi_{n}^{j}$ from $s_{0}^{j}$ provide the analogue of the vector functions $\tilde{\pi}_{k}^{j h}$ from sub-problems. The common goods prices are taken from the master simplex, prices of non-common goods come from sub-equilibrium solutions but are multiplied by a scalar $\lambda_{k}^{j}$ for each group $k$ such that prices of common goods are the same on the master simplex and at each sub-equilibrium problem. We write the vector $\left(\pi_{1}^{j}, \ldots, \pi_{n}^{j}\right.$, $\hat{\pi}^{1 j}, \ldots, \hat{\pi}^{K j}$ ) as $\hat{\pi}^{j}$ which we in turn normalize to sum to $D$. We note that the vector $\hat{\pi}^{j}$ contains all sub-equilibrium solution vectors for both common and non-common goods transformed by a scalar for each group; the relative prices of common and non-common goods for each group remain unaltered between subequilibria and the master problem. If a limit is approached for the case of a dense grid for which $c_{k}^{j^{*}}$ approaches zero for all $k$, the first $n_{0}$ entries of the normalized vector $\hat{\pi}^{j}$ will appoach zero.

For the $n_{0}$ dimensional master simplex we use the traditional GaleNikaido mapping to produce a continuous mapping of the simplex into itself whose fixed points along with associated sub-equilibria meet the required equilibrium conditions. We note that by Walras' Law and the features of noncommon goods, the value of excess demands for all common goods must equal zero. The mapping is given by the well known transformation of the excess demand functions for common goods evaluated at the vector $\hat{\pi}^{j}$ and summed over all groups 


$$
g_{i}\left(\pi_{1}^{j}, \ldots, \pi_{n_{0}}^{j}\right)=\frac{\pi_{i}^{j} / D+\max \left(0, \sum_{k=1}^{k}\left(\xi_{i}^{k}\left(\hat{\pi}^{j}\right)-w_{i}^{k}\right)\right)}{1+\sum_{i=1}^{n} \max \left(0, \sum_{k=1}^{k}\left(\xi_{i}^{k}\left(\hat{\pi}^{j}\right)-w_{i}^{k}\right)\right)} \quad\left(i=1, \ldots, n_{0}\right)
$$

The label $l\left(\delta_{0}^{j}\right)$ for vertices on the master simplex is determined as follows:

(i) If any element of $s_{0}^{j}$ is zero, $\ell\left(s_{0}^{j}\right)$ is the index value of the first zero entry.

(ii) If $\mathrm{s}_{0}^{j}$ is strictly positive, the label is determined as the first index 1 for which the image $g_{1}\left(\pi_{1}, \ldots, \pi_{n}^{j}\right)$ exceeds $\frac{1}{D}$ multiplied by the corresponding coordinate on $s_{0}^{j}$, (For $\left.i=1, \ldots, n_{0}\right)$.

\section{Demonstration of Equilibrium Conditions}

It remains to argue that a completely labelled simplex in the subdivision of $\mathrm{S}_{0}$ will be an approximation to an equilibrium of the entire general equilibrium model by a similar argument to that given in Scarf (1973) for a traditional general equilibrium model. There is a unique way to start the computational procedure on the master simplex as in Scarf's original algorithm and subsequently on each simplex in any sub-problem, and the Lemke-Howson no-cycling argument applies in exactly the same manner.

In order to argue that an approximation to an equilibrium for the entire problem will be found which becomes exact as the mesh corresponding to subdivisions of $s_{o}$ (and the corresponding simplices for sub-equilibrium problems) becomes finer and finer it remains to show that a completely labelled simplex in the subdivision of the master simplex will 'Imply that all equilibrium conditions must hold.

The demonstration of this uses the same procedure as with a traditional Gale Nika1do mapping where a fixed point under this mapping can be shown to 
Imply the equilibrium conditions for a pure trade economy. A completely labelled simplex in the subdivision of the master simplex in the limiting case of a dense grid yields a price vector at which all excess demands for common goods are nonpositive, common good prices are zero if any excess demand is strictly negative. A completely labelled simplex in the subdivision of each associated simplex for sub-problems ylelds a price vector at which excess demands for non-common goods are non-positive with non-common goods prices being zero if any excess demand is strictly negative. Both of these arguments use the property mentioned earlier that Walras Law together with the restriction that non-common goods cannot be traded across groups of consumers implies that the value of excess demands for common goods must equal zero, and similarly equals zero for each bloc of non-comon goods. The equilibrium price vectors at master and subequilibria are related by a scalar for each group and from the zero homogeneity of demand functions the prices of common and non-common goods at sub-equilibria can be transformed to be identical to those characterizing a master equilibrium. Thus both master and sub-equilibrium conditions hold at the same set of prices and an equilibrium to the entire model must have been determined.

IV. A Mumerical Example and Some Initial Computational Experience

Although we have described our procedure in terms of Scarf's original algorithm, the procedure can be easily adapted to extensions of..Scarf.'s algorithm due to Merrill (1971), vander Laan and Talman (1979) and others. We have adapted Merrill's algorithm to this procedure and solved some numerical examples by this method. A computational point of some significance with the application of a decomposttion procedure in such circumstances is the ability through a restart method to refine the grids for master and subsimplices at different speeds, an option not available with solution of the full problem. 
We have programmed, the decomposition procedure described above and solved a number of numerical examples both by this method and by solution of the corresponding full dimensional problem. While further imporvements in efficiency of coding may be possible, our initial experience suggests that if the decomposition structure is sufficiently strong, significant computational savings are possible if that structure is exploited.

We consider a sequence of economies each with 2 common goods and an increasing number of blocs of non-common goods. Each bloc of non-common goods is of dimensionality 2 and each group of consumers corresponding to a bloc is of size 2. We consider each consumer in each group to have a CES preference function defined over common goods and non-common goods for that group. As we add blocs the general equilibrium problem increases by 2 goods and 2 consumer groups in dimensionality. Our parameter sets involve preference weightings which differ between consumers (although not markedly), and endowments which in aggregate are similar for each group.

We solve the same problem by both a full solution method and the decomposition method described above. We use Merrill's algorithm for full solution. We then adapt Merrill's algorithm to the decomposition procedure by using an artificial layer for both master and subsimplices. The same initial guess is used in both procedures. The recent method developed by vander Laan and Talman could also be used and would almost certainly be quicker for both methods, probably by a common factor approximated by the range vander Laan and Talman suggest of 3-5. For comparative purposes the consistent use of Merrili's algorithm for full and decomposition solution seems to provide the indications we seek that the potential gains from a decomposition approach increase as the decomposition structure becomes more and more evident. 
Table 1 reports execution times as the sequence of economies extends by the addition of further consumer blocs. Increasingly improved relative performance by the decomposition procedure is evident as more blocs are added. We have not exploited the use of differential speeds of grid refinement between master and sub-problems which could yield further computational gains. Computational costs increase less sharply with the overall problem dimension with the decomposition method than under full solution; an approximation which does not seem too far off is that execution times under full solution are proportional to the third power of the number of blocs while the decomposition execution time is proportional to the third power of the total number of goods (which increases more slowly). Comparisons in terms of the number of replacement operations required are also possible although we have not reported them. While this experience is only suggestive and may not hold for other examples, it does indicate a potential computational gain through exploitation of decomposition structure.

V. Conclusion

In this paper we present a decomposition procedure for general equilibrium computation in models where a partition of the commodities into common and non-common goods is possible. The similarity of the computational method outlined in this paper to the Dantzig-Wolfe decomposition algorithm for the solution of linear programming is the ability to solve a large-scale general equilibrium problem through the separate solution of a sequence of smaller dimensional problems. Revisions to the master problem in Dantzig-Wolfe through coefficient generation are replaced by label generation for vertices on a master simplex.

Initial indications are that differences in computational cost between solution by the procedure described and full dimensional 


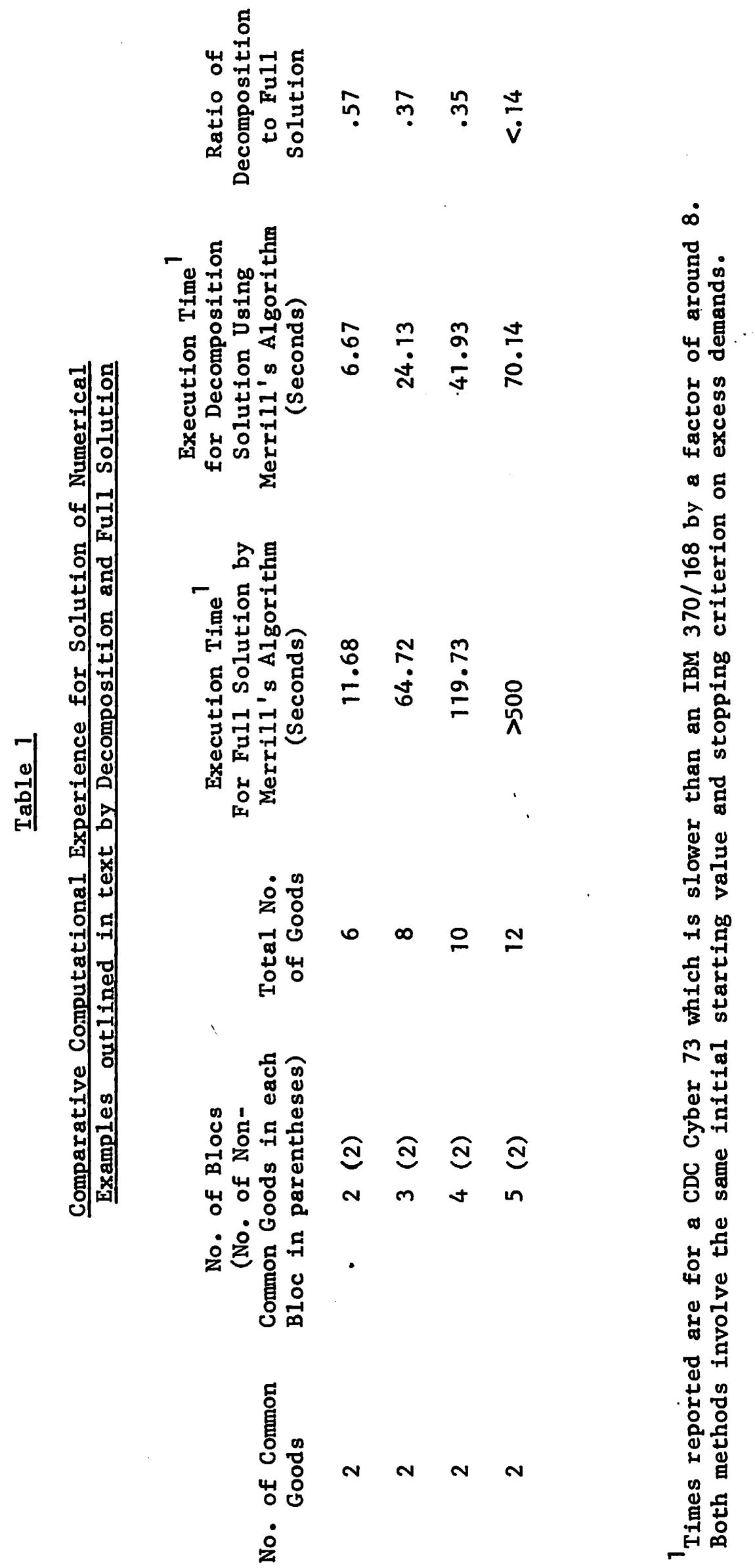


solution become increasingly significant as the decomposition structure becomes more pronounced and we report our experience in a comparative table. We suggest eventual application of this procedure may be possible to 'empirically' oriented large-scale general equilibrium models of world trade where the required structure is present. At least two such models incorporating traded and non-traded goods [Deardorff and Stern (1979), and Whalley (1979)] are in current use and others may follow in future developments. 


\section{References}

Arrow, K. J. and Hahn, F. H. General Competitive Analysis, Holden Day, San Francisco, 1971.

Dantzig, G. B. and Wolfe, P. "The Decomposition Algortthm for Linear Programs," Econometrica, 1961.

Deardorff, A. V. and Stern, R. M. "An Economic Analysis of the Effects of the Tokyo Round of Multilateral Trade Negotiations on the United States and the Other Major Industrialized Countries," MTN Studies No. 5, prepared for Subcommittee on International Trade, Committee on Finance, U.S. Senate, 96th Congress, Washington, D.C., 1979. Laan, G. Vander and Talman, A. J. J. "A Restart Algorithm for Computing Fixed Points Wi.thout an Extra Dimension," Mathematical Programming, 1979.

Merril1, 0. H., "Applications and Extensions of an Algorithm that Computes Fixed Points of Certain Non-empty Convex Upper Semi-Continuous Point to Set Mapping," Technical Report No. 71-7, Department of Industrial Engineering, University of Michigan, 1971.

Scarf, H., in collaboration with T. Hansen. The Computation of Economic Equilibria, Yale University Press, New Haven, 1973.

Shamir, Shlomo. "A Homotopy Fixed Point Algorithm with an Arbitrary Integer Refinement Factor," Working Paper SE-79R-2, Department of Engineering-Economic Systems, Stanford University, California, 1979.

Whalley, J. "General Equilibrium Analysis of EEC-US-Japanese Trade and Trade Distorting Policies: A Model and Some Initial Findings" to appear in a symposium on General Equilibrium and International Trade in Economie Applique. 
CTENTRE FOR THE STUDY OF INTERNATIONAL ECONOMIC PELATIONS

$$
\begin{gathered}
\text { University of Western Ontario } \\
\text { Working Papers }
\end{gathered}
$$

8001. Robson, Arthur J. OPEC VERSUS THE WEST: A ROBUST DUOPOLY SITUATION

8002. McMillan, John and Ewen McCann. WELFARE EFFECTS IN CUSTOMS UNIONS

8003. Leith, J. Clark. MONEY, THE BALANCE OF PAYMENTS, AND GOVEPNMENT DEBT IN A SMALL OPEN LDC: HAITI

8004. Mansur, Ashan and John Whalley. A DECOMPOSITION ALCORITHM FOR GFINERAL EQUILIBRIUM COMPUTATION WITH APPLICATION. TO INTERNATIONAL TRADE MODELS

8005. Schmid, Michael. OIL, EMPLOYMENT AND THE PRICE LEVEL: A MONETARY APPROACH TO THE MACROECONOMICS OF IMPORTED INIERMEDIATE GOODS UNDER FIXED AND FLEXIBLE RATES

8006. Markusen, James R. THE DISTRIBUTION OF GAINS FROM BILATERAL TARIFF REDUCTION

8007. Markusen, James R. TRADE AND THE GAINS FHOM TRADE WITH IMPERFECT COMPETITION

8008. Markusen, James R. and James R. Melvin. TRADE, FACTOR PRICES, AND THE GAINS FROM TRADE WITH INCREASING RETURNS TO SCALE 